\title{
Intermittency in Stochastically Perturbed Turbulent Models
}

\author{
L. Biferale, ${ }^{1,2}$ M. Cencini, ${ }^{3}$ D. Pierotti, ${ }^{4}$ and A. Vulpiani ${ }^{3,5}$
}

\author{
Received July 16, 1996; final February 3, 1997
}

\begin{abstract}
Random dynamical models obtained as a perturbation of the GOY (GledzerOhkitani-Yamada) shell model for three-dimensional turbulence are defined. Both static (time-independent) and dynamical scaling properties of the randomly perturbed model are studied. The random static-inviscid manifold, in contrast to the dynamical evolution, does not show intermittent scaling laws. This behavior is linked to the absence of large deviation in the random-map connecting fluctuations of velocities at different scales. The importance of inviscid conserved quantities on the intermittent statistics is discussed. Different random dynamical perturbations such that only energy is conserved in the inviscid and unforced limit are investigated. Intermittency is weakly affected by random perturbations.
\end{abstract}

KEY WORDS: Fully developed turbulence; intermittency; random maps; large deviations.

\section{INTRODUCTION}

The understanding of the small-scale statistics of three-dimensional fully developed turbulence is one of the main open problems of classical physics. The phenomenological theory of Kolmogorov (K41) and its sequent multifractal modification (see ref. 1 for a recent overview) give a qualitatively correct description of the main mechanisms acting at very high Reynolds numbers. One of the main goals is to understand the origin of scaling

\footnotetext{
${ }^{1}$ Dipartimento di Fisica, Università di Tor Vergata, I-00133 Rome, Italy.

${ }^{2}$ Istituto Nazionale Fisica della Materia, unità "Roma Tor Vergata," Rome, Italy.

${ }^{3}$ Dipartimento di Fisica, Università di Roma "la Sapienza," I-00185 Rome, Italy.

${ }^{4}$ Dipartimento di Fisica, Università dell' Aquila, I-67010 Coppito, L'Aquila, Italy.

${ }^{5}$ Istituto Nazionale Fisica della Materia, unità di Roma, Rome, Italy.
} 
laws for structure functions, $S_{p}(r)$, defined as the moments of velocity increments at scale $r$. Experiments ${ }^{(2)}$ show that structure functions scale as:

$$
S_{p}(r) \equiv\left\langle|\mathbf{v}(\mathbf{x}+\mathbf{r})-\mathbf{v}(\mathbf{x})|^{p}\right\rangle \equiv\left\langle\left|\delta_{r} v\right|^{p}\right\rangle \sim r^{\zeta(p)} \quad r=|\mathbf{r}|
$$

if $r$ is chosen in the inertial range, i.e. much smaller then the integral scale and much larger than viscous scales. There are strong experimental evidences that $\zeta(p)$ scaling exponents differ from the Kolmogorov dimensional result: $\zeta(p)=p / 3$. In the past, different multifractal random models for the energy transfer mechanism have been proposed ${ }^{(3,2)}$ All these models are able to fit with good accuracy the intermittent deviations from K41 theory. Deviations from $\mathrm{K} 41$ law are due to a non-trivial intermittent energytransfer mechanism from large to small scales. In the above approaches one basically uses phenomenological and probabilistic ideas without any direct link with the dynamical evolution given by the Navier-Stokes equations.

In order to go beyond the multifractal phenomenological descriptions one can approach the problem in two possible ways:

(i) analytical theory from first principles;

(ii) direct numerical simulations of the Navier-Stokes equations.

The difficulties of an analytical theory of fully developed turbulence are well known, and are common to all systems with strong nonlinearity and nongaussian behavior. ${ }^{(1)}$ Moreover, direct numerical simulations of the Navier-Stokes equations at very high Reynolds number are rather hard since the number $N$ of degrees of freedom necessary to describe the flow increases as $(R e)^{\alpha}, \alpha=9 / 4$ in the K41 theory.

On the other hand, since the existence of scaling laws, one can hope that the degrees of freedom in fully-developed turbulence are organized in a hierarchical way, so that simplified dynamical systems could be relevant for the description of small-scale properties. Simplified dynamical model for the energy cascade were proposed in the early seventies by Desnyansky and Novikov, ${ }^{(4)}$ and Gledzer. ${ }^{(5)}$ The first idea was to have a sort of closure scheme which is able to give the Kolmogorov law as a stable fixed point.

More recently, many authors proposed and studied chaotic shell models. ${ }^{(6,7,8,9)}$ The basic idea of shell models is to consider a discrete set of wave vector (shells) in the Fourier space and to construct a set of ordinary differential equations taking into account only few variables, typically one or two, for each shell. By denoting un the "velocity" variable in the shell $n$ with wave vector $k_{n}=k_{0} \lambda^{n}$, where $\lambda=2$ is the usual choice, we can think of $\left|u_{n}\right|$ as the velocity increments $\left|\delta_{r} v\right|$, at scale $r \sim k_{n}^{-1}$. The evolution equations for $u_{n}$ are built up according to some natural criteria: 
(i) the linear term of $u_{n}$ is given by $v k_{n}^{2} u_{n}$;

(ii) the nonlinear terms for $u_{n}$ are quadratic combinations of the form $k_{n} u_{n^{\prime}} u_{n^{\prime \prime}}$;

(iii) in absence of forcing and viscosity one has conservation of energy $\frac{1}{2} \sum_{n}\left|u_{n}\right|^{2}$ and the Liouville theorem, i.e. $\sum_{n} \partial \dot{u}_{n} / \partial u_{n}=0$, so that the volume in phase space is conserved;

(iv) the interactions among shells are local in $k$-space, i.e. $n^{\prime}$ and $n^{\prime \prime}$ are close to $n$.

The most studied shell model has been introduced by Ohkitani and Yamada who used a set of complex variables $\left(u_{1}, \ldots, u_{n}\right)$ satisfying the equations:

$$
\frac{d}{d t} u_{n}=-v k_{n}^{2} u_{n}+i k_{n}\left[a_{n} u_{n+1} u_{n+2}+\frac{b_{n}}{2} u_{n-1} u_{n+1}+\frac{c_{n}}{4} u_{n-1} u_{n-2}\right]^{*}+f_{n} \delta_{n, 4}
$$

where, in order to have energy conservation when $v=f=0$, one has:

$$
a_{n}=1, b_{n}=-\varepsilon \quad \text { and } \quad c_{n}=-(1-\varepsilon)
$$

with $n=1, \ldots, N$ and boundary conditions:

$$
b_{1}=b_{N}=c_{1}=c_{2}=a_{N-1}=a_{N}=0
$$

In the inviscid and unforced case one has, besides the energy conservation, another conservation law. For $\varepsilon<1$ there exists a helicity-like invariant:

$$
H_{\varepsilon}=\sum_{n}(-1)^{n} k_{n}^{\alpha(\varepsilon)}\left|u_{n}\right|^{2}
$$

while for $\varepsilon>1$ one has an enstrophy-like (positive-defined) invariant:

$$
\Omega_{\varepsilon}=\sum_{n} k_{n}^{\alpha(\varepsilon)}\left|u_{n}\right|^{2}
$$

where

$$
\alpha(\varepsilon)=-\ln _{2}|1-\varepsilon|
$$

Let us stress that for $\varepsilon=1 / 2$ one has that $H_{\varepsilon}$ is a sort of "shell model helicity" similar to the "true" helicity $H=\int(\mathbf{k} \times \mathbf{v}(\mathbf{k})) \cdot \mathbf{v}(\mathbf{k}) d \mathbf{k}$ conserved in the $3 \mathrm{~d}$ Euler equations. For the value $\varepsilon=5 / 4$ one has $\Omega_{\varepsilon}=\sum_{n} k_{n}^{2}\left|u_{n}\right|^{2}$, i.e., 
a quantity having the same dimension and form of the enstrophy. One can assume that the GOY model with such a value of $\varepsilon$ mimics the $2 \mathrm{~d}$ turbulence. ${ }^{(10)}$ We note that the number $N$ of shells necessary to reach the Kolmogorov scale is rather small: $N \sim \ln R e$. Therefore, one has a dynamical system with a moderate number of degrees of freedom and so it is possible to use methods and techniques of deterministic chaos. In particular, it is possible to study the intermittency corrections in great details and to relate the traditional statistical description (e.g., in term of structure functions and statistical properties of the energy dissipation) with the dynamical properties in the phase space (e.g., Lyapunov exponents and dimensions of the attractor). ${ }^{(6,7)}$

Detailed numerical studies show that the GOY model (2) with $\varepsilon=1 / 2$ is able to reproduce in a remarkable way many features observed in experiments and/or predicted by phenomenological models (e.g., the multifractal approach ${ }^{(7)}$ ). Among them, the most important one is the presence of an intermittent energy transfer leading to non-trivial scaling laws of structure functions. Structure functions for shell models are naturally defined by considering that on should describe velocity fluctuations at scale $r=1 / k_{n}$ :

$$
S_{p}\left(k_{n}\right)=\left\langle\left|u_{n}\right|^{p}\right\rangle \sim k_{n}^{-\zeta(p)}
$$

Numerical simulations of (2) with the choice of $\varepsilon$ parameter such as the second invariant coincides with the "helicity" $(\varepsilon=1 / 2)$ have the same quantitative degree of intermittency measured in real turbulent flows, i.e., the set of $\zeta(p)$ exponents measured from (8) coincides with the intermittent exponents found from Navier-Stokes evolution.

Another study ${ }^{(11)}$ concentrated on the static properties of the inviscid manifold of (2). GOY shell model has a K41-like static inviscid manifold. In ref. 11 the authors showed that the K41 static-scaling of Eq. (2) is stable under deterministic perturbation obtained by slightly modifying the original shell model equations (see next section). This result suggest that the origin of intermittency in shell models must be found in a non-trivial dynamical mechanism which intermittently drives away the trajectory from the static K41 manifold.

As for the intermittent dynamical properties of GOY model some authors ${ }^{(12,13,14)}$ conjectured that the agreement with the experimental data is strongly related to the existence of the second (helicity-like) invariant (5). In particular, in ref. 14 the authors claim that the intermittency corrections disappear if the second invariant is destroyed. They support this claim with the numerical computation of a modified shell model with non-local interactions. 
Even if intermittency in shell models can be strongly non-universal, we believe that it is certainly worthwhile to understand its origins. The dependency of intermittency on the non-linear term structure can help for highlighting the physical mechanisms responsible of the non-trivial energy transfer.

In this paper we analyze this issue by studying a class of shell models, without the second conservation law, obtained by stochastically perturbing the non-linear terms of the original GOY model.

Let us introduce a random version of the GOY model (2) where $a_{n}, b_{n}$ and $c_{n}$ are now random coefficients; in order to have the energy conservation for $v=f=0$ one has to impose the constraint:

$$
a_{n}+b_{n+1}+c_{n+2}=0
$$

In our random version we substitute (3) with:

$$
a_{n}(t)=1, b_{n}(t)=-\varepsilon_{n-1}(t), \quad \text { and } \quad c_{n}(t)=-\left(1-\varepsilon_{n-2}(t)\right)
$$

where $\varepsilon_{n}(t)$ are independent stochastic processes. It is easy to realize that if $\varepsilon_{n}(t)$ are not fixed in time there are not other conservation laws besides energy, in the inviscid non-forced limit case $v=f=0$. In the following we assume that $\varepsilon_{n}(t)=\frac{1}{2}+\delta \varepsilon_{n}(t)$ where $\delta \varepsilon_{n}$ is a gaussian stochastic process such that

$$
\overline{\delta \varepsilon_{n}(t)}=0 \quad \text { and } \quad \overline{\delta \varepsilon_{n}(t) \delta \varepsilon_{n^{\prime}}\left(t^{\prime}\right)}=\sigma_{n}^{2} \delta_{n, n^{\prime}} \exp \left(-\frac{\left|t-t^{\prime}\right|}{\tau_{n}}\right)
$$

In the limit $\tau_{n} \rightarrow \infty$ one has a quenched disorder.

Another stochastic shell model for the energy cascade, rather different from our model, has been proposed an studied in ref. 15 .

In section 2 we shall discuss the static properties of the shell model in the inertial range in the limit of quenched disorder. This problem can be reformulated in terms of products of random matrices and can be treated analytically. It is rather interesting that, in spite of the disorder, one has not anomalous scaling, in agreement with the results obtained in the analogous deterministic problem. ${ }^{(11)}$ This behaviour is connected to the absence of large-deviations, at least in the sense leading to anomalous scaling behaviour, in the probability distribution density of the variable describing fluctuations of shell-velocities between adjacent scales.

Section 3 is devoted to the study of the scaling of the structure functions obtained from the dynamical rhodel $(2)$, with choice $(10,11)$ for different values of $\sigma$ and $\tau_{n}$, e.g., $\tau_{n}=$ const. or $\tau_{n} \sim k_{n}^{-2 / 3}$ (which has the same scaling properties of the eddy turn over times). The differences from 
the usual GOY model are small and indicate a strong robustness of intermittent corrections against stochastic perturbation of the non-linear terms of model. This result suggests that the origins of intermittency cannot be only connected to the presence of a second non-positive defined invariant.

\section{STATIC MANIFOLD PROPERTIES}

In this section we study the scaling properties of the inviscid-staticmanifolds of model (2) with random coefficients.

By noticing that model (2) has an inviscid-static-manifold with K41 scaling, on $u_{n} \sim k_{n}^{-1 / 3}$, it is natural to try to understand intermittent corrections starting from the inviscid-static behaviour of the dynamical evolution (2). This question was already investigated in ref. 11 for the original GOY model. The idea is to recover from the non-linear part of the equations a multiplicative process which gives the scaling properties of the static solution. In the "infinite" Reynolds number limit $(v=f=0)$ the static manifold of Eq. (2) is:

$$
u_{n+2} u_{n+1}-\frac{\varepsilon}{2} u_{n+1} u_{n-1}-\frac{(1-\varepsilon)}{4} u_{n-1} u_{n-2} \equiv 0
$$

which can be written as a one-dimensional complex ratio-map for the variables $w_{n}=u_{n} / u_{n-3}$ :

$$
w_{n}=\frac{\varepsilon}{2}+\frac{1-\varepsilon}{4 w_{n-1}}
$$

The map describes a "static-cascade," connecting velocity fluctuations at different scales through a multiplicative process. ${ }^{6}$ It is difficult to quantify apriori how many properties of this map are connected to the full timedependent dynamical evolution of model (2).

Forward iterations of map (13) converges toward the K41 fixed point, $w^{\mathrm{K} 41}=1 / 2$, for any $\varepsilon \in[0,2]$.

In ref. 11 non-trivial (chaotic) trajectories for successive iteration of the ratio-map were obtained by adding a deterministic small perturbation given by another possible shell model, the Novikov-Desnyansky model. ${ }^{(4)}$ Scaling laws of the static manifold can be obtained as a product of successive ratios, $q_{n}=u_{n} / u_{n-1}$. Structure functions are defined as:

$$
S_{n}^{(p)}=\left\langle\prod_{k=1}^{n}\left|q_{k}\right|^{p}\right\rangle \approx\left\langle\prod_{k=1}^{n}\left|w_{k}\right|^{p / 3}\right\rangle \sim k_{n}^{-\zeta(p)}
$$

\footnotetext{
${ }^{6}$ This is exactly in the same spirit of phenomelogical random models for the energy cascade as the Random- $\beta$-Model ${ }^{(3)}$ or the p-model. ${ }^{(2)}$
} 
where $w_{n}=q_{n} q_{n-1} q_{n-2}$. In ref. 11 authors showed that the extended ratiomap, obtained by using a linear combination of GOY and NovikovDesnyansky models, develops deterministic chaos, and $\langle\ldots\rangle$ in (14) can be intended as averages over different initial conditions. The remarkable fact presented in ref. 11 is that, although the presence of chaos, structure functions (14) are asymptotically Kolmogorov-like, i.e., intermittent deviations are absent. This absence of intermittency can be connected to the absence of large-deviations in the probability distribution density of the variable obtained by multiplying $n$ successive map iterations. Indeed, expression (14) can be written as:

$$
S_{n}^{(p)}=\left\langle k_{n}^{\gamma(n) p / 3}\right\rangle \sim k_{n}^{-\zeta(p)} \quad \text { with } \quad \gamma(n)=\frac{1}{n} \sum_{k=1}^{n} \log _{2}\left|w_{k}\right|
$$

and, therefore, non-trivial and intermittent exponents $\zeta(p) \neq p / 3$ can be obtained only if the probability distribution of instantaneous scaling exponent, $\Pi(\gamma(n))$, satisfies large deviations theorem. ${ }^{(1)}$ Large deviations theorem for the normalized sum of $n$ stochastic variables, $\gamma(n)$, says that the probability, $\Pi(\gamma(n))$, that $\gamma(n)$ assumes values in the range $[\gamma, \gamma+d \gamma]$ is given, for $n \rightarrow \infty$, by ref. 1 :

$$
\operatorname{Prob}\left\{\frac{1}{n} \sum_{k=1}^{n} \log _{2}\left|w_{k}\right| \in[\gamma, \gamma+d \gamma]\right\} \propto \exp (-n S(\gamma))
$$

where $S(\gamma)$ is the Cramer function and has properties similar to the $f(\alpha)$ of the multifractal description. ${ }^{(16)}$ Let us notice that even if equation 16 may not be true, large-deviations could still hold in its general form: ${ }^{(17)}$

$$
\operatorname{Prob}\left\{\frac{1}{n} \sum_{k=1}^{n} \log _{2}\left|w_{k}\right| \in[\gamma, \gamma+d \gamma]\right\} \propto \exp \left(-a_{N} s(\gamma)\right)
$$

where $a_{n}$ is some increasing diverging sequence; pure scaling behaviour is obtained only in the case where $a_{n} \sim n$. In the following we shall adopt the term large-deviation only for the pure scaling case. From (15) and (16) we can define scaling exponents in the limit of an infinite-extended inertial range by performing a saddle point estimate:

$$
\zeta(p)=-\max _{\gamma}\left(\gamma \frac{p}{3}-\frac{S(\gamma)}{\ln 2}\right)
$$

K41 scaling, $\zeta(p)=p / 3$, means that large deviation theorem is not applicable, i.e., (16) does not hold. A similar behaviour can be found in 
sporadic maps, a class of maps where marginally unstable fixed-point dominates the asymptotic dynamical behaviour. ${ }^{(18)}$ In shell-model ratiomap this would mean that K41 stable manifolds (or a submanifold of it) should play a marginally attractive role for the dynamics, leading to a failure of large deviations theorem and, as a direct consequence, to nonintermittent scaling behaviour.

In the following we show that the absence-of-large-deviations found in ref. 11 for deterministic perturbation of (13) is still valid even in the case of stochastic perturbation provided that the constraint imposed by non-linear dynamical conservation of energy is not broken.

Inviscid energy conservation imposes a weak structural constraint for the choice of the free parameters in the non-linear terms of (2).

A stochastic quenched perturbation to the non-linear static manifold can be introduced by imposing that:

$$
a_{n}=1, b_{n}=-\varepsilon_{n-1}, c_{n}=-\left(1-\varepsilon_{n-2}\right)
$$

where $\varepsilon_{n}$ is a stochastic process. Let us notice that in the GOY model (2) with choice (19) conservation of energy is still insured in the inviscid limit, while the second inviscid invariant is $\sum_{n=1}^{N} A_{n}\left|u_{n}\right|^{2}$, where now the $\left\{A_{n}\right\}$ depend on the $\left\{\varepsilon_{n}\right\}$ realization.

The static inviscid manifold is now defined as:

$$
u_{n+2} u_{n+1}-\frac{\varepsilon_{n}-1}{2} u_{n+1} u_{n-1}-\frac{\left(1-\varepsilon_{n-2}\right)}{4} u_{n-1} u_{n-2}=0
$$

which leads to the random ratio-map for $w_{n}=u_{n} / u_{n-3}$ :

$$
w_{n}=\frac{\varepsilon_{n}}{2}+\frac{1-\varepsilon_{n-1}}{4 w_{n-1}}
$$

In what follows most of our analysis have been done by choosing $\varepsilon_{n}$ to be identically, independently and uniformly distributed in the interval $[\bar{\varepsilon}-W, \bar{\varepsilon}+W]$, where the central value $\bar{\varepsilon}$ has been fixed to $\bar{\varepsilon}=1 / 2$. The parameter $W$ controls the strength of the stochastic perturbation. We have also checked that by slightly varying the probability distribution function of $\varepsilon_{n}$ none of the results hereafter reported change.

Structure functions for this random-static manifold are defined as in (14) with the only difference that now averages $\langle\ldots\rangle$ must be intended over different realization of the random process. Different $\varepsilon_{n}$ realizations mimic the different paths followed by the energy transfer from large to small scales in a turbulent flow. 


\subsection{Structure Functions Scaling}

We have computed scaling properties of the structure functions defined in terms of the random ratio-map up to order 12 , at varying $W$ in the range $[0,2]$. Averages have been done over $\approx 10^{6}$ different realizations of $\left(\varepsilon_{1}, \ldots, \varepsilon_{n}\right)$ sequences, and by considering a huge number of fragmentation steps, $n \approx 200$.

In Fig. 1 we show a log-log plot of the structure functions. As it is possible to see, there is a clean straight line behaviour in perfect agreement with the Kolmogorov non-intermittent prediction $\zeta(p)=p / 3$ (Fig. 1a).
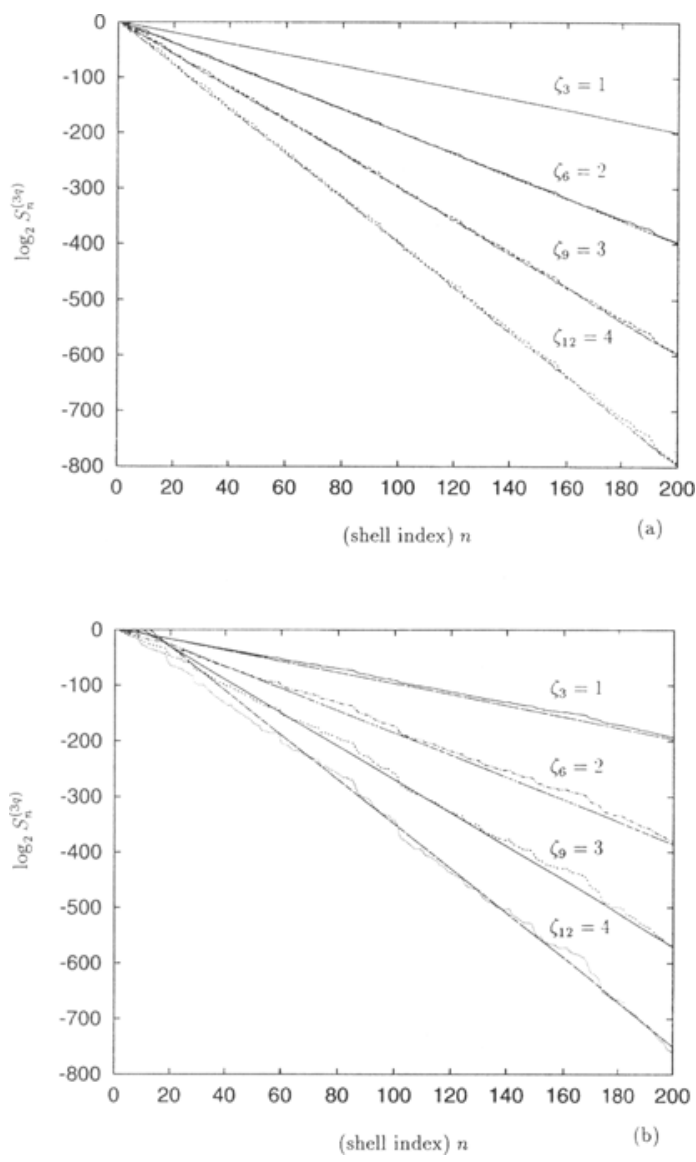

Fig. 1. (a) Log-log plot of the structure functions $S_{n}^{(3 q)}$ os $k_{n}$ for the random ratio-map (21) with $\bar{\varepsilon}=0.5$ and $W=0.5$ (solid lines) -1.5 (dashed lines), for $q=1, \ldots, 4$. (b) Same of Fig. (a) but with $W=2.0$. The straight lines indicate the K4l scaling. 
Fig. $1 \mathrm{~b}$ shows that for large value of $W$ the $\mathrm{K} 41$ scaling is reached only in the limit $n \rightarrow \infty$ (see discussion in Section 2.2). Therefore, we can safely conclude that, also by using stochastic perturbation to the GOY static manifold, large deviations are absent. This results is stable for different choices of $W \in[0,2]$ and $\bar{\varepsilon} \in[0,2]$.

Since the stochastic nature of map (21), the absence of large deviations is now much more peculiar than the analogous result in ref. 11 for the deterministic case.

Let us now investigate this problem by using product of random matrices theory. ${ }^{(19)}$ By writing $w_{n}=\varphi_{n} / \varphi_{n-1}$ we have:

$$
\varphi_{n}=\frac{\varepsilon_{n}}{2} \varphi_{n-1}+\left(\frac{1-\varepsilon_{n-1}}{4}\right) \varphi_{n-2}
$$

which can be rewritten as:

$$
\mathbf{z}_{n}=A(n) \mathbf{z}_{n-1}
$$

where:

$$
\mathbf{z}_{n}=\left(\begin{array}{c}
\varphi_{n} \\
\varphi_{n-1}
\end{array}\right), \quad A(n)=\left(\begin{array}{cc}
\frac{\varepsilon_{n}}{2} & \frac{1-\varepsilon_{n-1}}{4} \\
1 & 0
\end{array}\right)
$$

Let us stress that matrices $A(n)$ are correlated. Indeed, the energy constraint introduces a dependency of $A(n)$ entries on two successive $\varepsilon$ realizations. From (23) and (14) we have:

$$
S_{n}^{(q)} \equiv\left\langle\prod_{k=1}^{n}\left|w_{k}\right|^{q}\right\rangle=\left\langle\prod_{k=1}^{n}\left|\frac{\varphi_{k}}{\varphi_{k-1}}\right|^{q}\right\rangle=\left\langle\left|\frac{\varphi_{n}}{\varphi_{0}}\right|^{q}\right\rangle \sim\left\langle\left(\frac{\left|\mathbf{z}_{n}\right|}{\left|\mathbf{z}_{0}\right|}\right)^{q}\right\rangle
$$

Expression (25) connects the asymptotic properties of the product $w_{n} w_{n-1} \cdots w_{1}$ with the asymptotic properties of the random matrices product $A(n) A(n-1) \cdots A(1)$. In order to characterize the asymptotic behaviour of a random matrix products is useful to introduce the concept of generalized Lyapunov exponents. ${ }^{(3)}$ Given a sequence of random matrix $X(1), X(2), \ldots, X(N)$ the Oseledec and Furstenberg theorem states that the limit

$$
\lim _{N \rightarrow \infty} \frac{1}{N} \ln \left\|P_{N}\right\|=\lambda_{1}, \quad \text { with } \quad P_{N}=\prod_{k=1}^{N} X(k)
$$


exists with probability 1 and $\lambda_{1}$ is the maximum Lyapunov exponent. Generalized Lyapunov exponents are defined as:

$$
L(q)=\lim _{N \rightarrow \infty} \frac{1}{N} \ln \left\langle\left\|P_{N}\right\|^{q}\right\rangle
$$

and measure large-fluctuations around the mean value $\lambda_{1}=(d / d q)$ $\left.L(q)\right|_{q=0}$. In particular, in absence of large fluctuations we have $L(q)=$ $\lambda_{1} q$. By using (25) we can connect the scaling exponents, $\zeta_{3 q}$, with the generalized Lyapunov exponents, $L(q)$ :

$$
\left\langle\left|\mathbf{z}_{n}\right|^{q}\right\rangle \sim\left\langle\left\|P_{n}\right\|^{q}\right\rangle \sim \exp (n L(q))
$$

with $P_{n}=\prod_{k=1}^{n} A(k)$. Comparing (28), (25) and (14) we find:

$$
\zeta_{3 q}=-\frac{L(q)}{\ln 2}
$$

There are some techniques for evaluating the $L(q)$ exponents ${ }^{(19)}$ in the case of products of independent random matrices. Recently, in ref. 20 it has been shown how to generalize the replica-trick to the case of products of matrices generated by a Markov process with a finite number of states. In order to apply this result we have slightly modified the probability distribution function of $\varepsilon$ by choosing $\varepsilon_{n}$ to assume only a finite number, say $M$, of possible values in the interval $[\varepsilon-W, \varepsilon+W]$. With this choice for the random variable $\varepsilon$ we have the $M^{2}$ possible matrices:

$$
A^{(\alpha, \beta)}=\left(\begin{array}{cc}
\frac{\varepsilon^{(\alpha)}}{2} & \frac{1-\varepsilon^{(\beta)}}{4} \\
1 & 0
\end{array}\right) \quad \text { with } \quad \alpha, \beta=1, \ldots, M
$$

which correspond to the $M^{2}$ states of the Markov chain.

Energy conservation imposes that if $A(n)=A^{(\alpha, \beta)}$ than $A(n+1)$ must be one of the $M$ matrices $A^{(\gamma, \alpha)}$ with $\gamma=1, \ldots, M$. Introducing the matrix $T(i, j)$ with $i, j=1,2, \ldots, M^{2}$ which defines the Markov conditional probability of transition between two states, one can apply the replica-trick method for Markov chains (see appendix 1 and ref. 20) and find:

$$
L(q)=\ln \left(y_{q}\right)
$$

where $y_{g}$ is the eigenvalue with maximum absolute value of $Y_{q}$ and $Y_{q}$ is a matrix $M^{2} 2^{q} \times M^{2} 2^{q}$ obtained from $T$ and $A$ (see appendix 1). Because of the size of the matrix $Y_{q}$ there are obvious numerical limitations in the 
application of this methods for large values of $q$ and $M$. Relation (31) is valid for any positive and even $q$, while for odd $q$ 's it is correct only if $Y_{q}$ has non-negative entries.

We have applied this method to compute the scaling exponents. We found that for $\varepsilon$ chosen to assume discretized values in $[0,1]$ all scaling exponents are strictly Kolmogorov-like, within computer-precision. This analytical result confirm robustness of static properties of the GOY model under stochastic perturbation. As long as non-linear terms are chosen such as to dynamically conserve energy, there are not any intermittent deviations in the scaling properties of the static manifold.

In order to understand the origin of the absence of intermittency we have relaxed the constraint (19). we have therefore taken matrices $A(n)$ :

$$
A(n)=\left(\begin{array}{cc}
\frac{\varepsilon_{n}}{2} & \frac{1-\varepsilon_{n}^{\prime}}{4} \\
1 & 0
\end{array}\right)
$$

where now $\varepsilon_{n}$ and $\varepsilon_{n}^{\prime}$ are i.i.d. variables. Replica-trick for the $A(n)$ uncorrelated matrices is now very simple. If $\varepsilon_{n} \in[0,1]$ the matrix $A$ has non-negative elements and it is easy to see that $L(1)$ is the logarithm of the maximum eigenvalue of the matrix:

$$
\bar{A}=\left(\begin{array}{cc}
\bar{\varepsilon} & \frac{1-\bar{\varepsilon}}{4} \\
1 & 0
\end{array}\right)
$$

Therefore we obtain:

$$
\zeta_{3}=-L(1)=-\frac{\max \left\{\left|v_{1}\right|,\left|v_{2}\right|\right\}}{\ln 2}
$$

where:

$$
v_{1}=\frac{1}{2} \quad \text { and } \quad v_{2}=\frac{1-\bar{\varepsilon}}{2}
$$

It is interesting to notice that in the above case (34) has the "Kolmogorov solution" $\zeta_{3}=1$. On the other hand, higher $q$ values give $L(q)$ exponents which deviate from the Kolmogorov straight line behaviour $L(q)=q$. In Fig. 2, we show the log-log plot of structure functions obtained for this 


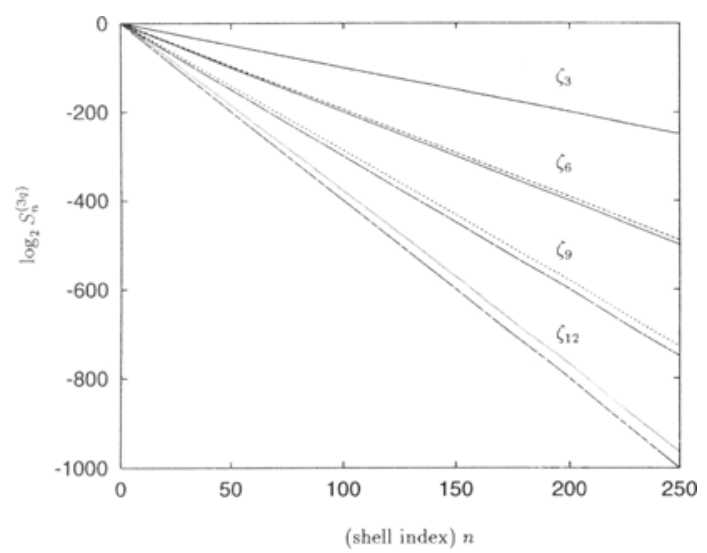

Fig. 2. Log-log plot of the structure functions $S_{n}^{(3 q)}$ vs $k_{n}$ (dashed lines) for the random ratio-map with uncorrelated matrices (32). Solid lines are the K41 scaling.

uncorrelated case. For $q>1$ one has small intermittent deviations from the Kolmogorov prediction.

This result indicates that the absence of intermittency in randomratio-map must originate from the correlations introduced by the energyconservation constraint chosen in the original GOY model.

Let us anticipate that, as we will show in Section (3), dynamical evolution of the energy-conserving random-model is nevertheless intermittent. Dynamical evolution shows different scaling properties than the staticmanifold.

This must be connected, as for the deterministic case, ${ }^{(11)}$ to complex dynamical mechanisms introducing intermittent deviations from the K41 manifold in the time evolution of the trajectory in the phase space.

\subsection{Probability Distribution Density}

The absence of intermittency can be interpreted from (15) and (16) as a lack of large deviation properties for the probability distribution of $\gamma(n)=\frac{1}{n} \sum_{k=1}^{n} \log _{2}\left|w_{k}\right|$. Let us call $\Pi_{n}(\gamma) d \gamma$ the probability that $\gamma(n)$ falls in the interval $[\gamma, \gamma+d \gamma]$. Large deviation theory states that the in the limit of $n \rightarrow \infty$ there exist a unique limiting curve for the probability distribution:

$$
\lim _{n \rightarrow \infty} \frac{1}{n} \ln \frac{\prod_{n}(\gamma)}{\prod_{n}^{\max }}=-S(\gamma)
$$


where we have normalized $\Pi_{n}(\gamma)$ with its maximum value in order to eliminate pre-asymptotic terms. For small deviations from the mean value $\gamma_{m}=\langle\gamma\rangle$ the central limit theorem predicts the usual parabolic shape:

$$
S(\gamma)=\frac{\left(\gamma-\gamma_{m}\right)^{2}}{2 \sigma^{2}} \quad \text { with } \quad \sigma^{2}=\lim _{n \rightarrow \infty}\left\langle n\left(\gamma-\gamma_{m}\right)^{2}\right\rangle
$$

In Fig. 3 we show that at varying $\varepsilon$ and $W$ we find instances where the limiting curve (36) is not defined and/or instances where the central limit theorem is not verified. Indeed, in Fig. $3 \mathrm{a}$ the probability distribution functions decrease faster than the exponential of $n$; in Fig. $3 \mathrm{~b}$ the large
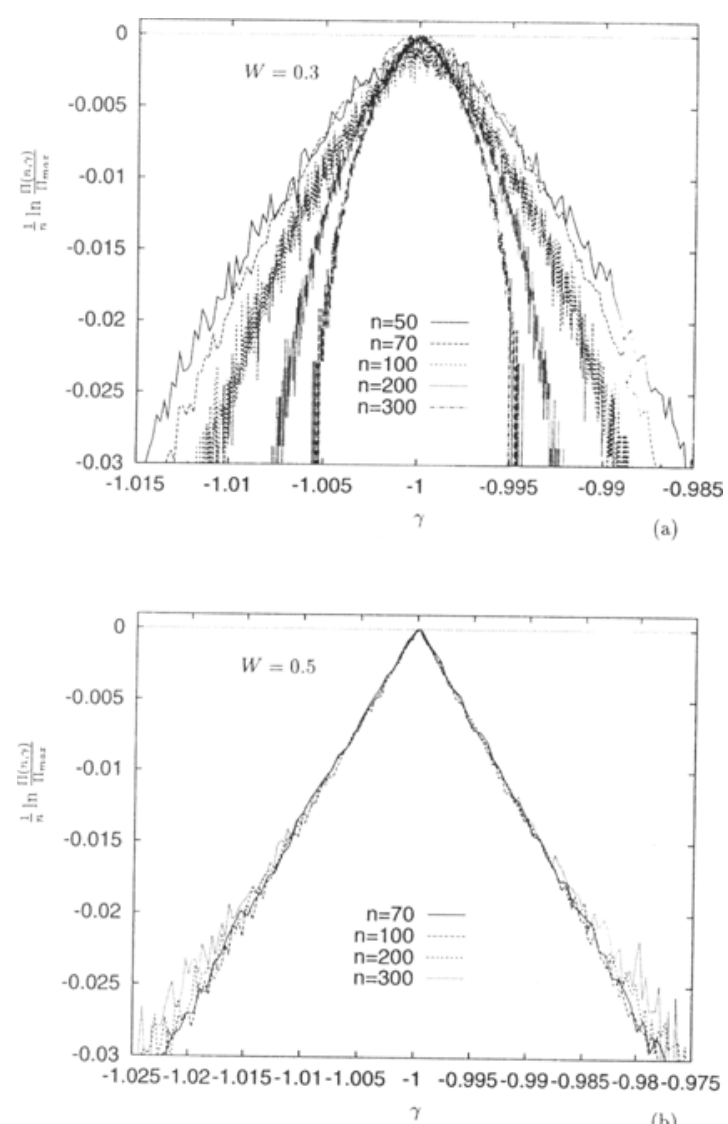

Fig. 3. (a) Plot of $1 / n \ln \left(\prod_{n}(\gamma) / \Pi_{n}^{\max }\right) v s \gamma(n)$ for the map (21) with $\bar{\varepsilon}=0.5$ and $W=0.3$. (b) The same as in Fig. (a) with $W=0.5$. 
deviation theorem holds but there is a strong violation of the central limit theorem and one has the atypical behaviour $S(\gamma) \approx\left|\gamma-\gamma_{m}\right|$.

We can fit all data by supposing a most general form for the probability distribution function:

$$
P(\gamma) \propto \exp \left(-n^{\alpha} \frac{\left|\gamma-\gamma_{m}\right|^{\beta}}{C}\right)
$$

where the constants aand $\beta$ depend on $\varepsilon$ and $W$. For $\varepsilon \in[0,2]$ and for any value of $W$ we have the Kolmogorov mean value $\gamma_{m}=-1$. From (38) we can estimate structure as:

$$
\left\langle\prod_{k=1}^{n}\left|w_{k}\right|^{q}\right\rangle=\left\langle k_{n}^{q \gamma(n)}\right\rangle=\int k_{n}^{q \gamma} P(\gamma) d \gamma \propto \int k_{n}^{q \gamma} k_{n}^{-n^{\alpha}\left|\gamma-\gamma_{m}\right|^{\beta /} / C \ln 2} d \gamma \sim k_{n}^{-\zeta_{3 q}}
$$

which can be evaluated in a saddle point approximation $k_{n} \rightarrow \infty$, giving:

$$
-\zeta_{3 q}=\max _{\gamma}\left\{q \gamma-n^{\alpha-1} \frac{\left|\gamma-\gamma_{m}\right|^{\beta}}{C \ln 2}\right\}
$$

In the instances where we found $\alpha>1$ and $1 \leqslant \beta<2$, we can deduce that the asymptotic limit must be $\zeta(q)=q / 3$ and the leading corrections to this non-intermittent behaviour are given by:

$$
\delta \zeta_{3 q}=\zeta_{3 q}-q \sim O\left(\frac{1}{n^{(\alpha-1) /(\beta-1)}}\right)
$$

Let us notice that in the cases where the large deviations theorem holds $(\alpha=1)$, like in Fig. 3b, we have a strong violation of the central limit theorem.

Indeed the Cramer function, $S(\gamma)$, is not differentiable at the maximum and therefore it is not possible to use the saddle point estimate (40). ${ }^{(21)}$ It is easy to realize that in this case the asymptotic scaling properties must be Kolmogorov-like.

Both effects here discussed, i.e., absence of large deviations $(\alpha \neq 1)$ and failure of central limit theorem $(\beta<2)$, are certainly due to non-trivial correlations introduced in the stochastic process by the energy conservation constraint. The relevance of this constraint for the presence of a "static-intermittency" would suggest that conserved quantities play an important role in the generation of complex energy-transfer mechanism. In the following section we investigate the dynamical evolution of the randomly perturbed GOY model given by $(2)$ with the choices $(10,11)$. 


\section{DYNAMICAL PROPERTIES}

In this section we discuss the scaling properties of the stochastic GOY model introduced in Section 1. We use the following choice for the random coefficients: $\varepsilon_{n}(t)=1 / 2+\delta \varepsilon_{n}(t)$, where $\delta \varepsilon_{n}(t)$ is obtained from a linear Langevin equation:

$$
\frac{d \delta \varepsilon_{n}}{d t}=-\frac{\delta \varepsilon_{n}}{\tau_{n}}+\sqrt{\frac{2 \sigma_{n}^{2}}{\tau_{n}}} \eta_{n}
$$

where $\eta_{n}$ are independent white noises, i.e. gaussian processes with

$$
\overline{\eta_{n}(t)}=0, \quad \overline{\eta_{n}(t) \eta_{m}\left(t^{\prime}\right)}=\delta_{n m} \delta\left(t-t^{\prime}\right)
$$

It is easy to see that this simple stochastic process produces the (11). The numerical algorithm for $(42)$ is:

$$
\delta \varepsilon_{n}(t+\Delta t)=A_{n} \delta \varepsilon_{n}(t)+B_{n} \eta_{n}(t)
$$

with

$$
A_{n}=e^{-\Delta t / \tau_{n}}, \quad B_{n}=\sigma_{n} \sqrt{1-e^{-2 \Delta i / \tau_{n}}}
$$

An easy direct computation shows that the rule (44) gives (11) if $t / \Delta t$ and $t^{\prime} / \Delta t$ are integers. Let us note that $A_{n} \simeq 1-\Delta t / \tau_{n}$ and $B_{n} \simeq \sigma_{n} \sqrt{2 \Delta t / \tau_{n}}$. In our numerical experiments we used different choices for $\sigma_{n}$ and $\tau_{n}$ :

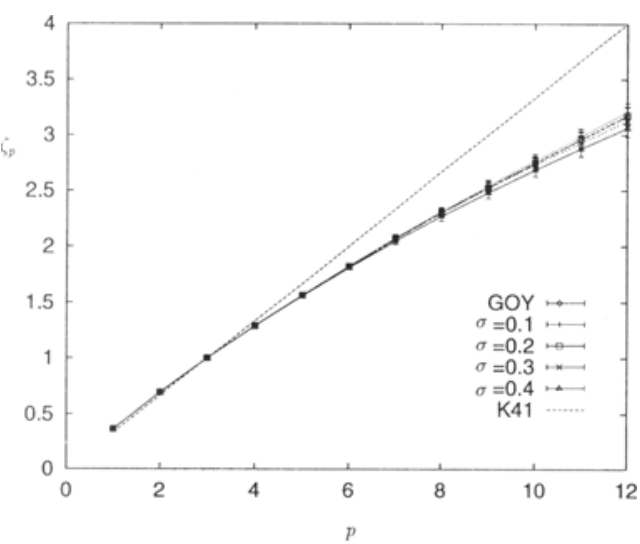

Fig. 4. Anomalous scaling exponents for the case $\bar{\varepsilon}=0.5$ in which all shells are perturbed. Different curves correspond to different values of the noise amplitude: $\sigma=0.1, \ldots, 0.4$, while $\tau \approx 10^{-5} \tau_{\text {eddy }}$ in each shell, where $\tau_{\text {eddy }}$ is the eddy turn-over time of the first shell. The straight line corresponds to the Kolmogorov scaling $\zeta_{p}=p / 3$. 
(a) perturbations on each shell: $\sigma_{n}=\sigma, n=1, \ldots, N$; with equal correlation time for each shell: $\tau_{n}=\tau$;

(b) the same of (a) but with $\tau_{n}$ proportional to the eddy turn-over time at scale $l_{n} \sim k_{n}^{-1}: \tau_{n}=T k_{n}^{-2 / 3}$.

We performed the numerical computations in the above ways with different values of $\sigma, \tau$ and $T$ in order to test the "structural stability" of the structure functions anomalous scaling.

All the results have been obtained with $N=19$ number of shells, $v=10^{-6}$, $k_{0}=2^{-4}, a=5 \times 10^{-3}(1+i)$ and $d t=3 \times 10^{-4}$, integrating the equations
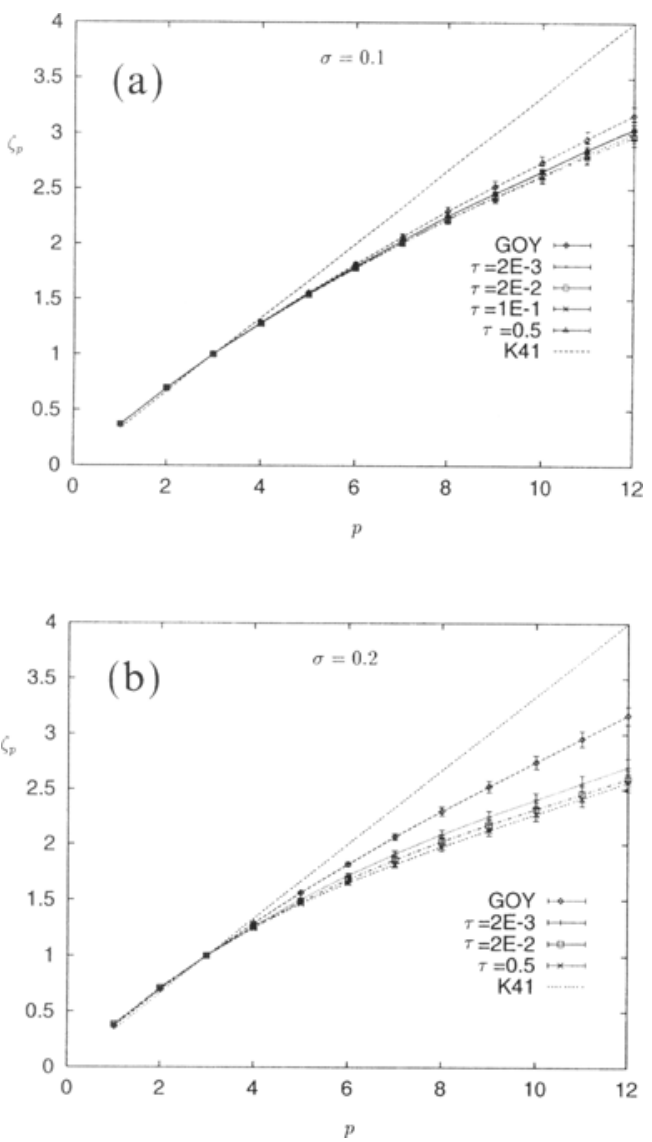

Fig. 5. (a) Same as in Fig. 4 but with $\sigma=0.1$ and different choices of the correlation time $\tau: \tau / \tau_{\text {eddy }} \in\left[2 \cdot 10^{-3}, 5 \cdot 10^{-1}\right]$. (b) Same as in Fig. (a) with $\sigma=0.2$. 
by a slaved Adams-Bashforth algorithm. Time integrations vary up to $10^{4}$ eddy-turnover times of the largest scale, $\tau_{\text {eddy }}$. Several runs have been made with $N=22, v=10^{-7}, k_{0}=2^{-4}, f=5 \times 10^{-3}(1+i)$ and $d t=10^{-4}$ to check the dependence of the results on the Reynolds number. No relevant differences have been detected.

In Figs. 46 we show some typical results for the $\zeta_{p}$ 's. It is evident that the stochastic-GOY-model scaling exponents are quite different from the $\mathrm{K} 41$ value $\zeta_{p}=p / 3$. Moreover, the scaling laws for the stochastic model are close to those of the non-random model.

This results are well understood in the case in which the noise correlation times are well below the lowest inertial eddy turn-over time (Fig. 4). The fluctuations of the random perturbation-no matter of their amplitude - have weak influence on the system. The dynamical behaviour is expected to be similar to that of the usual model with coefficients equal to the mean values of the noise (see Fig. 4).

On the other hand, we get some appreciable quantitative differences from the anomalous exponents of the non-random model when we use correlation times comparable to the eddy turn-over times and large noise amplitude. In this case the increased intermittency (see Fig. $5 \mathrm{~b}$ and compare it with Fig. 5a) is probably due to the increased amplitude of the fluctuations in forward and backward energy transfer. Let us notice that with such choice of noise-time-correlation there will be scales with quenched noise and scales with annealed noise. The "structural stability" of the model is properly tested using noise correlation times of type (b). In this case, characteristic perturbation times are of the same order of the eddy-turn-over

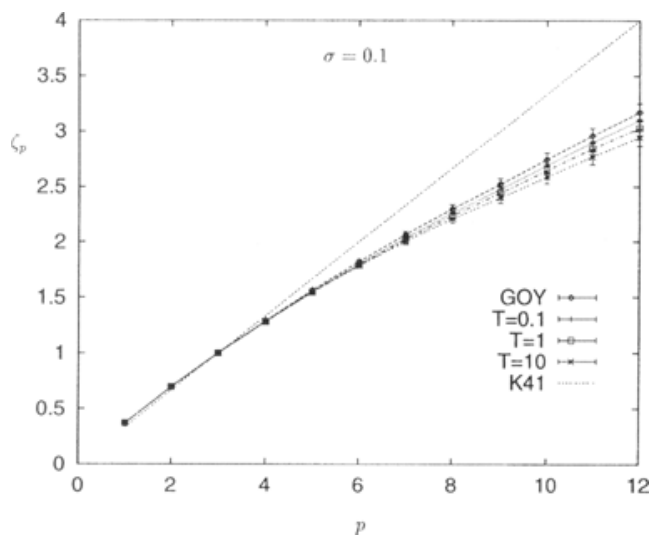

Fig. 6. $\zeta_{p}$ obtained in the case $\bar{\varepsilon}=0.5$ of $\sigma=0.1$ and a shell dependent $\tau: \tau_{n}=T\left(k_{n} / k_{0}\right)^{-2 / 3}$, with $T \approx 0.1,1,10 \tau_{\text {eddy }}$. 
times for each shell. As one can see in fissure $d$, there are not appreciable differences with the non-random case.

\section{CONCLUSIONS}

A class of randomly perturbed GOY models for turbulent flows has been introduced and studied. Both static and dynamical scaling properties have been investigated analytically and numerically.

Static and dynamical properties shows very different behaviour with respect to the dependence from inviscid conserved quantities. In particular, static properties have always Kolmogorov-like scaling unless inviscid energy conservations is relaxed. Dynamical properties are intermittent and do not show strong dependencies from the presence of conserved quantities.

As for the random-static inviscid manifold, we found that the Kolmogorov-like scaling is connected to the failure of large-deviation and/or central limit theorem for the random variables obtained from the map describing random fluctuations of velocities at successive scales. The absence of large deviations is also connected to the physical constraint of inviscid energy conservation which, must be imposed in order to stay as close as possible to the original Navier-Stokes equations.

On the other hand, the dynamical evolution of the ordinary equations (2) with random coefficients (10) is intermittent. The degree of intermittency seems to be weakly affected by the details of the random perturbation. By noticing that as soon as the model is randomly perturbed one of the two original inviscid invariant (the helicity-like invariant) is destroyed, we conclude that intermittency cannot be only related to the competition between the two invariants (energy and helicity). Weak non-universality as a function of the perturbation structure are observed only in the case when characteristic times of the random perturbation are comparable (or larger) than the inertial range eddy-turn-over times.

Robustness of the dynamical energy transfer mechanism against random perturbations is the main result of this paper.

\section{A. REPLICA TRICK FOR MARKOV PROCESS}

The replica trick ${ }^{(19)}$ is one of the most used tool for calculating generalized Lyapunov exponents in the case of correlated and uncorrelated product of random matrices. The uncorrelated case is particularly simple 
and it is based on the asymptotic realization for the product, $P_{N}$, of $N$ random matrices $X_{i}$ :

$$
L(q)=\lim _{N \rightarrow \infty} \frac{1}{N} \ln \left\langle\left\|P_{N}\right\|^{q}\right\rangle=\lim _{N \rightarrow \infty} \frac{1}{N} \ln \left\langle\left|\operatorname{Tr} P_{N}\right|^{q}\right\rangle
$$

For even and positive $q$ 's we have:

$$
\left\langle\left(\operatorname{Tr} P_{N}\right)^{q}\right\rangle=\operatorname{Tr}\left[\left\langle P_{N}^{\otimes q}\right\rangle\right]=\operatorname{Tr}\left[\overline{X^{\otimes q}}\right]^{N}
$$

where

$$
X^{\otimes q}=\overbrace{X \otimes X \otimes \cdots \otimes X}^{q \text { times }}
$$

is the $q$ th direct product, and with $\overline{(\cdot)}$ we intend the matrix obtained after averaging on the stochastic process. From (46) and (47) we obtain:

$$
L(q)=\ln \left(x_{q}\right)
$$

which holds for even $q$ 's and where $x_{q}$ is the eigenvalue of $\overline{X^{\otimes q}}$ with maximum modulus. For odd $q$ 's it holds only if matrix $X$ has non-negative entries.

In the case of correlated random matrices we have that the first equality in relation (47) still holds, while the second relation is obviously broken. In the case of Markov process with a finite number of state is still possible to overcome this difficulty. ${ }^{(20)}$ Using the notation of Section 2.1 we define:

$$
B(i)=A_{i}^{\otimes q}
$$

and

$$
T(i, j)=\frac{1}{M} \delta_{\alpha(M-1)+\beta, \gamma(M-1)+\alpha}, \quad \gamma=1, \ldots, M
$$

where $T(i, j)$ defines the Markov conditional probability of transition from state $i=\alpha(M-1)+\beta$ to $j=\gamma(M-1)+\alpha$ (using these labels for the states the energy conservation constraint is automatically satisfied). Therefore we have:

$$
\begin{aligned}
\left\langle P_{N}^{\otimes q}\right\rangle & =\left\langle\prod_{n=1}^{N} A^{\otimes q}(n)\right\rangle \\
& =\sum_{\left\{i_{\eta}\right\}} P\left(i_{1}\right) B\left(i_{1}\right) T\left(i_{1}, i_{2}\right) B\left(i_{2}\right) T\left(i_{2}, i_{3}\right) \cdots B\left(i_{N-1}\right) T\left(i_{N-1}, i_{N}\right) B\left(i_{N}\right)
\end{aligned}
$$


where with $P\left(i_{1}\right)$ we indicate the probability that the initial matrix coincide with the state $i_{1}$, and $\left\{i_{\eta}\right\}$ represents the sum over all the possible realizations. By writing

$$
Y_{q}(\alpha, i ; \beta, j)=T(i, j)(B(j))_{\alpha, \beta}
$$

it is easy to realize that (51) corresponds to the usual matrix product and therefore:

$$
\left\langle\left(P_{N}^{\otimes q}\right)_{\alpha, \beta}\right\rangle=\sum_{i_{N}} \sum_{i_{0}} Y_{q}^{N}\left(\alpha, i_{0} ; \beta, i_{N}\right)
$$

Using (47) and (53) we can finally write:

$$
L(q)=\lim _{N \rightarrow \infty} \frac{1}{N} \ln \left\langle\left\|P^{\otimes} q_{N}\right\|\right\rangle=\lim _{N \rightarrow \infty} \frac{1}{N} \ln \operatorname{Tr}\left|Y_{q}^{N}\right|=\ln \left|y_{q}\right|
$$

where $\left|y_{q}\right|$ is the eigenvalue with maximum modulus of the matrix $Y_{q}$. For example in the case $M=2$, matrix $Y_{q}$ is:

$$
Y_{q}=\left(\begin{array}{cccc}
\frac{1}{2} A_{1}^{\otimes q} & 0 & \frac{1}{2} A_{3}^{\otimes q} & 0 \\
\frac{1}{2} A_{1}^{\otimes q} & 0 & \frac{1}{2} A_{3}^{\otimes q} & 0 \\
0 & \frac{1}{2} A_{2}^{\otimes q} & 0 & \frac{1}{2} A_{4}^{\otimes q} \\
0 & \frac{1}{2} A_{2}^{\otimes q} & 0 & \frac{1}{2} A_{4}^{\otimes q}
\end{array}\right)
$$

\section{REFERENCES}

1. U. Frisch, Turbulence, (Cambridge University Press, UK, 1995).

2. C. M. Meneveau and K. R. Sreenivasan, "The multifractal nature of turbulent energy dissipation" J. Fluid. Mech. 224:429 (1991).

3. R. Benzi, G. Paladin, G. Parisi and A. Vulpiani, "On the multifractal nature of fully developed turbulence and chaotic systems," J. Phys. A 17:3521 (1984).

4. V. N. Desnyansky and E. A. Novikov, "Evolution of turbulence spectra to self-similar regime," Izv. Akad. Nauk SSSR Fiz. Atmos. Okeana 10:127 (1974).

5. E. B. Gledzer, "System of hydrodynamic type admitting two quadratic integrals of motion," Sov. Phys. Dokl. 18:216 (1973).

6. M. Yamada and K. Ohkitani, "The inertial subrange and non-positive Lyapunov exponents in fully developed turbulence," Prog. Theor. Phys. 79:1265 (1988); M. Yamada and K. Ohkitani, "Temporal intermittency in the energy cascade processes and local Lyapunov analysis in fully developed model turbulence," Prog. Theor. Phys. 81:329 (1989).

7. M. H. Jensen, G. Paladin and A. Vulpiani, "Intermittency in a cascade model for three dimensional turbulence," Phys. Rev. A 43:798 (1991).

8. D. Pisarenko, L. Biferale, D. Courvoisier, U. Frisch and M. Vergassola, "Further results on multifractality in shell models," Phys. Fluids A 5:2533 (1993). 
9. R. Benzi, L. Biferale and G. Parisi, "On intermittency in a cascade models of turbulence," Physica D 65:163 (1993).

10. E. Aurell, G. Boffetta, A. Crisanti, P. Frick, G. Paladin and A. Vulpiani, "Statistical mechanics of shell models for 2D-turbulence," Phys. Rev. E 50:4705 (1994).

11. L. Biferale, M. Blank and U. Frisch, "Chaotic Cascades with Kolmogorov 1941 Scaling," J. of Stat. Phys. 75:781 (1994).

12. L. Kadanoff, D. Lohse, J. Wang, R. Benzi, "Scaling and Dissipation in the GOY shell model," Phys. Fluids 7:617 (1995).

13. L. Biferale and R. Kerr, "On the role of inviscid invariants in shell models of turbulence," Phys. Rev. E 52:6113 (1995); R. Benzi, L. Biferale, R. Kerr and E. Trovatore, "Helical shell models for three dimensional turbulence," Phys. Rev. E 53:3541 (1996).

14. O. Gat, I. Procaccia and R. Zeitak, "The breakdown of dynamical scaling and intermittency in a cascade model of turbulence," Phys. Rev. E 51:1148 (1994).

15. J. Eggers, "Intermittency in dynamical model for turbulence," Phys. Rev. A 46:1951 (1992).

16. B. B. Mandelbrot, "Random multifractals: negative dimensions and the resulting limitations of the thermodynamic formalism," Proc. R. Soc. Lond. A 434:79 (1991).

17. R. S. Ellis, Entropy Large-Deviations and Statistical Mechanics, (Springer, Berlin 1985).

18. P. Gaspard and X-J Wang, "Sporadicity: Between periodic and chaotic dynamical behaviors," Proc. Natl. Acad. Soc. USA 85:4591 (1988); X-J Wang, "Statistical physics of temporal intermittency," Phys. Rev. A 40:6647 (1989).

19. A. Crisanti, G. Paladin and A. Vulpiani, Products of Random Matrices, (Springer Verlag, Berlin 1993).

20. M. J. de Oleiveira and A. Petri, "Generalized Lyapunov Exponents for Products of Correlated Random Matrices," Phys. Rev. E 53:2960 (1996).

21. A. Crisanti, G. Paladin, H. J. Sommers and A. Vulpiani, "Replica trick and fluctuation in disordered systems," J. Phys, 2:1325-1332 (1992). 